\title{
Physiology and pathophysiology of pleural fluid turnover
}

\author{
G. Miserocchi
}

Physiology and pathophysiology of pleural fluid turnover. G. Miserocchi. CERS Journals Ltd 1997.

ABSTRACT: The pleural space contains a tiny amount $\left(\approx 0.3 \mathrm{~mL} \cdot \mathrm{kg}^{-1}\right)$ of hypooncotic fluid $\left(\approx 1 \mathrm{~g} \cdot \mathrm{dL}^{-1}\right.$ protein $)$. Pleural fluid turnover is estimated to be $\approx 0.15$ $\mathrm{mL} \cdot \mathrm{kg}^{-1} \cdot \mathrm{h}^{-1}$. Pleural fluid is produced at parietal pleural level, mainly in the less dependent regions of the cavity. Reabsorption is accomplished by parietal pleural lymphatics in the most dependent part of the cavity, on the diaphragmatic surface and in the mediastinal regions. The flow rate in pleural lymphatics can increase in response to an increase in pleural fluid filtration, acting as a negative feedback mechanism to control pleural liquid volume. Such control is very efficient, as a 10 fold increase in filtration rate would only result in a $15 \%$ increase in pleural liquid volume. When filtration exceeds maximum pleural lymphatic flow, pleural effusion occurs: as an estimate, in man, maximum pleural lymph flow could attain 30 $\mathrm{mL} \cdot \mathrm{h}^{-1}$, equivalent to $\approx 700 \mathrm{~mL} \cdot \mathrm{day}^{-1}(\approx \mathbf{4 0 \%}$ of overall lymph flow).

Under physiological conditions, the lung interstitium and the pleural space behave as functionally independent compartments, due to the low water and solute permeability of the visceral pleura. Pleural fluid circulates in the pleural cavity and intrapleural fluid dynamics may be represented by a porous flow model. Lubrication between lung and chest wall is assured by oligolamellar surfactant molecules stratified on mesothelial cells of the opposing pleurae. These molecules carry a charge of similar sign and, therefore, repulse each other, assuring a graphite-like lubrication.

Eur Respir J., 1997; 10: 219-225.
Istituto di Fisiologia Umana, Università degli Studi, Milano, Italy

Correspondence: G. Miserocchi Istituto di Fisiologia Umana

Via Mangiagalli 32

20133 Milano

Italy

Keywords: Fluid dynamics

lung interstitium

lung microvessels

lymphatics

micropuncture

Received: November 21995

Accepted after revision July 311996
A review on pleural space is certainly most welcome, for the simple reason that on opening a textbook of physiology the concepts concerning pleural fluid turnover date back to 1927 [1]. The same comment, in fact, applies in general to microvascular water and solute exchange. This appears particularly misleading, considering the major advances achieved in the field over the last 70 yrs. Around the turn of the 19th century, STARLING and TuBBy [2] interpreted microvascular fluid and solute exchange as resulting from the balance between hydraulic and colloidosmotic pressures. This concept is still valid today, but the complete formulation of transmicrovascular exchange has become much more complex because water crosses biological membranes more easily than large solutes (namely, plasma proteins) do [3]. In fact, different equations describe water and solute fluxes [4].

As a result, the general model of transcapillary fluid exchange still taught to students, based on fluid filtration at the arteriolar end of the capillary bed and reabsorption at the venular end, appears rather simplistic. Due to the different permeability to water and solutes, one could predict on a mathematical basis that such a model would lead to a progressive increase in interstitial protein concentration over time [3, 4], a condition that cannot be confirmed experimentally.

Similarly, the old hypothesis claiming that pleural fluid filters at parietal level and is reabsorbed through the visceral pleura [1] would imply that pleural liquid protein concentration would keep increasing with age, but again there are no indications that this occurs. The existence of partial restriction to the movement of large solutes, compared to that of water molecules, posed scientists the major problem of explaining how interstitial volume and protein concentration are kept fairly steady. Ideologically, this led to re-evaluation of lymphatics as the major route for interstitial fluid drainage. In fact, since lymphatics do not sieve proteins, they leave the interstitial protein concentration unaltered the latter depending only upon the sieving properties of the filtering membrane. Accordingly, the description of interstitial fluid homeostasis under steady state conditions is now being explained as a balance between capillary filtration and lymphatic absorption. Major validation of this model has come from the accumulating experimental data over the past 30 yrs concerning interstitial tissues [4] and serous spaces [5]. Interestingly, since the pioneering, work of Starling and TubBy [2] the pleural space has been used as a useful experimental model to study the interaction between microvascular filtration and lymphatic drainage.

This article presents an integrated view of pleural fluid turnover, based on data gathered from experimental studies on animals over the last 15 yrs. The situation in man is, unfortunately, still poorly defined; yet, where possible, 
extrapolations will be made in an attempt to relate the present state of knowledge to human pleural pathophysiology.

\section{The pleural compartments}

The pleural space, like other serous cavities of the body, may be considered an enlarged tissue space. In fact, unlike a common interstitial space, it presents a higher ratio of free fluid to solid tissue volume. Solid volume may be represented by cells being present in the pleural fluid and microvilli of mesothelial cells. The pleural space actually contains a tiny amount of fluid, ( $\approx 0.3 \mathrm{~mL} \cdot \mathrm{kg}^{-1}$ body mass, with a low protein concentration $\left(\approx 1 \mathrm{~g} \cdot \mathrm{dL}^{-1},[6,7]\right)$; the latter appears a peculiar feature considering that, in physiological conditions, the pressure of the pleural fluid is subatmospheric. In fact, when a subatmospheric pressure is found in other tissues, like the lung interstitium for example, protein concentration increases and, furthermore, it is well-known that an opposite condition, namely tissue hyperhydration, causes a decrease in interstitial protein concentration.

Figure 1 is a simplified schema of pleuropulmonary compartments. Considering the anatomical arrangements, it appears that five compartments are involved: the parietal systemic microcirculation; the parietal interstitial space; the pleural cavity itself; the lung interstitium; and the visceral microcirculation (either systemic from bronchial artery or pulmonary). The membranes separating such compartments are: the capillary endothelium (on parietal and visceral side); and the parietal and the visceral mesothelia. The lymphatics provide drainage of the interstitial spaces but also of the pleural cavity, as they open directly on the parietal pleura (lymphatic stomata). Stomata, in rabbits and sheep [8-11], are frequently grouped in clusters and connect to an extensive network of submesothelial lacunae $[11,12]$. They range in density from 100 stomata $\cdot \mathrm{cm}^{-2}$ on the intercostal

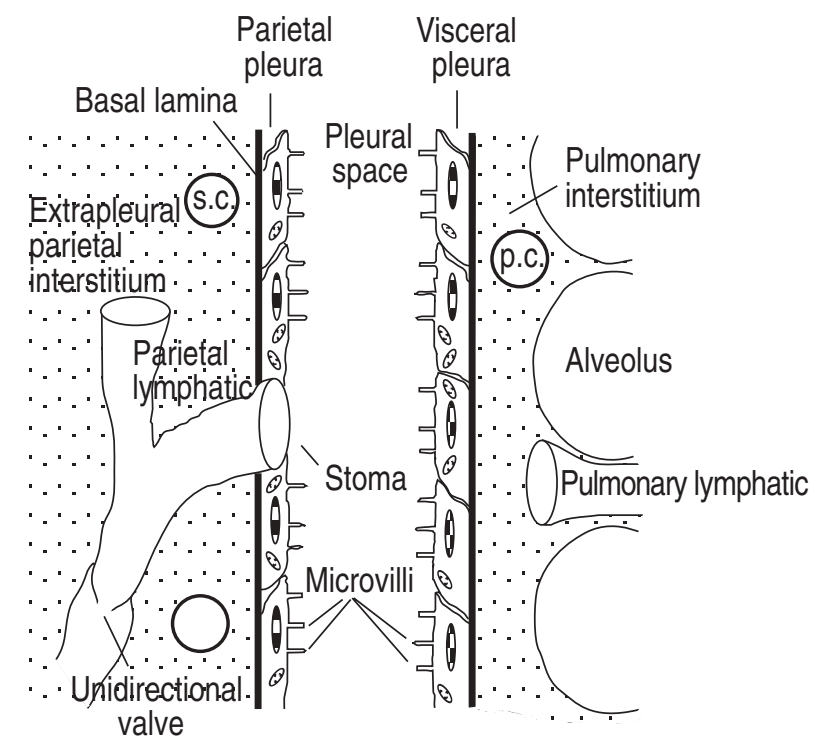

Fig. 1. - Schema of the morphofunctional design of pleural space; s.c.: systemic capillary; p.c.: pulmonary capillary. surface to $8,000 \cdot \mathrm{cm}^{-2}$ on the diaphragm and their diameter averages $1 \mu \mathrm{m}$ (range $<1-\sim 40 \mu \mathrm{m})$ in size. A similar anatomical disposition was seen for the peritoneal cavity. Lacunae in man have not been definitely demonstrated, although their existence is probable. Furthermore, they were found in mammals as large as sheep [13].

Mesothelial cells are only about $4 \mu \mathrm{m}$ thick [11], and connect to each other by tight junctions on the luminal side and by desmosomes on the basal portion of the intercellular junction [11].

Microvilli 1-3 $\mu \mathrm{m}$ long are seen on mesothelial cells, varying in density from 2 to 30 per $\mu \mathrm{m}^{2}$, and trap high concentrations of glycoproteins and hyaluronic acid [11, 14]. The thickness of the pleurae is quite variable among species: in animals with thin pleurae (dogs, rabbits and cats [15]), the thickness of the submesothelial interstitium is equal for parietal and visceral pleura, averaging $20 \mu \mathrm{m}$; however, in animals with thick pleurae (sheep, pig, horses and humans), it is about five times thinner in the parietal compared to the visceral pleura, where it can attain about $100 \mu \mathrm{m}[13,14,16]$.

It might be useful to recall briefly the concepts concerning fluid and plasma protein flux across biological membranes. All acceptable description of water flux (normally indicated as $\mathrm{J}_{\mathrm{v}}$ ) between two compartments labelled 1 and 2 is given by the revised Starling law:

$$
\mathrm{J}_{\mathrm{v}}=\mathrm{Kf}\left[\left(\mathrm{PH}_{1}-\mathrm{PH}_{2}\right)-\sigma\left(\pi_{1}-\pi_{2}\right)\right]
$$

where $\mathrm{Kf}$ is the filtration coefficient, $\mathrm{PH}$ and $\pi$ are the hydraulic and colloidosmotic pressures, and $\sigma$ is the solute reflection coefficient of the membrane. The coefficient, $\sigma$, is a number varying from 0 to 1 . For $\sigma=1$, the radius of the solute (we consider plasma proteins) is larger than that of the pores of the membrane and, therefore, no solute flux can occur through the membrane. For $\sigma=0$, the radius of the pores of the membrane is large enough to allow solute to cross the membrane. For $0<\sigma<1$, there is partial restriction to solute movement. The description of solute flux is rather difficult, as it occurs partly via the water flux and partly down a diffusion concentration gradient [3].

\section{The old hypothesis on pleural fluid turnover}

In 1927, based on the Starling hypothesis of fluid exchange, NeErgard [1] proposed the hypothesis that pleural fluid turnover is entirely dependent upon the difference between hydraulic and colloidosmotic pressure. Although provocative and relatively unchallenged for over half a century, this model appears today simplistic and untenable, as it neglects the existence of interstital spaces, the permselectivity to water and solutes, and the existence of pleural lymphatics. NeERGard [1] reasoned that pleural fluid filters at parietal level because hydraulic pressure in systemic microcirculation exceeds colloidosmotic pressure; conversely, fluid is reabsorbed at visceral level because in the pulmonary microcirculation the opposite is true. This hypothesis was developed by Agostoni et al. [17], who found that the difference between hydraulic and colloidosmotic pressure in the pulmonary capillaries was large enough to account for 
a)

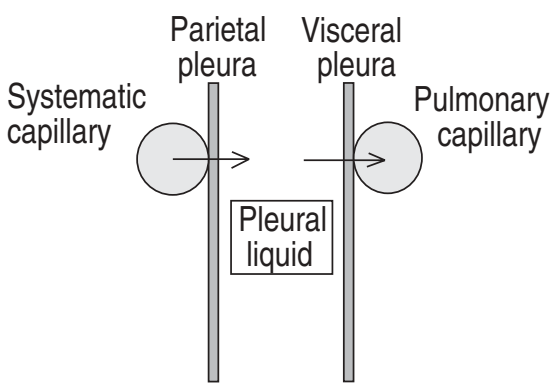

b) Parietal Visceral

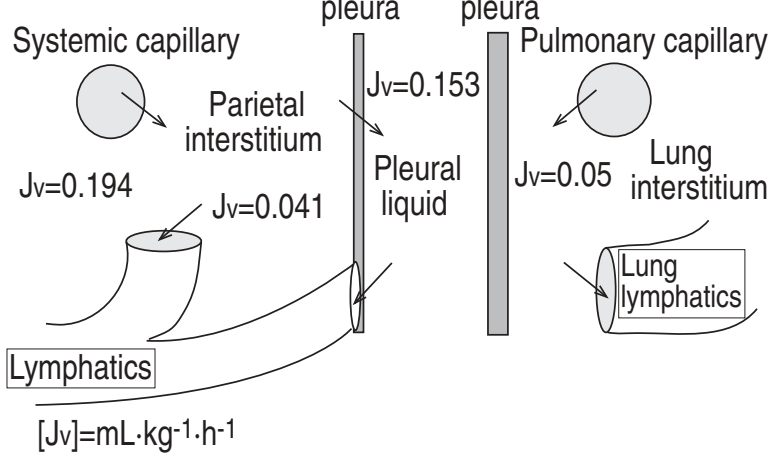

Fig. 2. - a) Neergard's hypothesis (1927) [1]. The old hypothesis concerning pleural fluid turnover, which neglects the existence of interstitial spaces and pleural lymphatics. b) the present view of pleural fluid turnover, based on the available experimental database gathered with minimally invasive techniques in the rabbit. The pleural space and the pulmonary interstitium are, under normal conditions, two functionally separate compartments, fluid filters from the microcirculation and is drained by the lymphatics. Subatmospheric hydraulic pressures are set by the action of the lymphatic pump. The figure shows water flux under steady state conditions. Solute (plasma protein) flux has been omitted. Although figures will vary in man, the overall situation is unlikely to be qualitatively different based on anotomofunctional considerations.

the subatmospheric pressure of the pleural liquid. This old hypothesis (fig. 2a) is no longer valid today based on the present state of knowledge. In fact, a thorough analysis of fluid (and solute) flux has required the development of sophisticated techniques to measure hydraulic and colloidosmotic pressures in the various pleural compartments $[18,19]$, to estimate water and solute permeability coefficients [7] of the membranes separating the compartments, and, finally, to measure pleural lymphatic flow in conditions very close to the physiological ones [20-23]. The whole mosaic of knowledge is fairly complete in rabbits, where most of the techniques were developed by preserving the integrity of the pleural space, and the available information has made it possible to develop a model of pleural fluid turnover that can be extended to animals as large as sheep and is compatible with pathophysiological observations in man.

\section{Pleural fluid filtration}

From experimental determination of variables and coefficients shown in the Starling equation [5, 7], it appears that pleural fluid is filtered at parietal pleural level from systemic microvessels to the extrapleural interstitium and from there into the pleural space down a relatively small pressure gradient (fig. $2 b$ ). The average filtration rate decreases with increasing animal size and ranges $\sim 0.1-0.02 \mathrm{~mL} \cdot \mathrm{kg}^{-1} \cdot \mathrm{h}^{-1}$, from rabbits to dogs. It is important to recall that, in order to derive indications on pleural fluid turnover, one must carry out experiments in conditions close to the physiological one that is characterized by two main features: namely, a small pleural liquid volume and subatmospheric pleural liquid pressures, such as those occurring during spontaneous breathing. In experimental models implying large pleural effusions and mechanical ventilation [24, 25], the database is difficult to interpret as it does not reflect a steady state situation.

Comparative studies (with animals ranging from a few gram to $50 \mathrm{~kg}$ body mass) reveal that pleural fluid turnover (normalized to body mass) decreases with increasing size [5]. In fact, it was found that with increasing mass: 1) pleural liquid pressure (considered at the level of the right atrium) becomes more negative; and 2) pleural fluid volume (normalized to body mass) and protein concentration decrease. Thus, it appears that, with increasing body mass, the tendency develops for a greater "dehydration" of the pleural space. At the extreme of this scale, anatomical records indicate that in animals as large as elephants, the pleural cavity is obliterated.

From the biophysical standpoint, the parietal mesothelium can be modelled as a membrane with few but large pores: this is reflected by a low $\sigma$ value $(\approx 0.3)$ but also by a low solute permeability coefficient [7]. This feature makes it possible to sieve proteins efficiently, so that the protein concentration of pleural fluid is rather low (about $1 \mathrm{~g} \cdot \mathrm{dL}^{-1}$ ), smaller than the protein concentration in the extrapleural parietal interstitium (about 2.5 $\left.\mathrm{g} \cdot \mathrm{dL}^{-1}[7]\right)$. Extrapolation to humans is difficult. Furthermore, in humans, there is the suggestion that some filtration may occur from the visceral pleura, as its blood supply stems from the systemic circulation where functional hydrostatic pressure is higher compared to pulmonary circulation; this fact however, may be of no relevance as the key variable in this respect is pulmonary interstitial pressure. The latter was directly measured in rabbits, by using the micropuncture technique through the pleural window approach, and was found to be rather subatmospheric $\left(\approx-10 \mathrm{cmH}_{2} \mathrm{O}[26]\right)$. The same technique made it possible to describe the pulmonary microvascular pressure profile [27]. Based on these findings, it appears that fluid normally filters from pulmonary microvessels to the lung interstitium. If pulmonary interstitial and microvascular pressures in humans were similar to those measured in rabbits, no gradient should be present to cause fluid filtration through the visceral pleura.

\section{Pleural fluid drainage}

Absorption flows through the visceral pleura are normally negligible, so that, under normal conditions, the pleural space and the pulmonary interstitium are two functionally separate compartments. This should also be true in man, given the great thickness of the visceral pleura, implying a low water and solute permeability. Most pleural fluid drainage ( $\approx 75 \%$ ), estimated from clearance of labelled proteins in rabbits and dogs, occurs through parietal pleural lymphatics [22, 23]. Controversial 
data on pleural lymph flow were gathered in sheep, where the same group of investigators found on different occasions that pleural lymphatics would drain either $<1 \%$ [25] or $\approx 70 \%$ of pleural fluid [24].

Pleural lymphatics are able to generate a subatmospheric pressure [21] of about $-10 \mathrm{cmH}_{2} \mathrm{O}$. Furthermore, they can increase the flow rate by about 20 fold in response to an increase in pleural liquid volume following an increase in pleural fluid filtration [20]. Lymphatic activity is pulsatile in nature, due partly to myogenic rhythmic contraction of the smooth muscles of the lymphatic walls (intrinsic activity), and partly to tissue pressure oscillations related to respiratory movements (extrinsic mechanism). The two mechanisms account for about 40 and $60 \%$, respectively, of total pleural lymphatic flow under physiological conditions, the stroke volume for each initial lymphatic stoma being of the order of $1 \times 10^{-6} \mu \mathrm{L} \cdot$ stoma [22]. Some features of the drainage are peculiar, as a greater lymphatic drainage occurs in the lowermost parts of the cavity [28], over the diaphragmatic surface and in the mediastinal regions [18]. Since filtration and absorption sites are different, this implies that pleural fluid circulates within the pleural space (see below under "Intrapleural fluid dynamics").

Figure 2a presents the old hypothesis of pleural fluid turnover, whilst the present model based on experimental results is shown in figure $2 \mathrm{~b}$. Fluid filters from the parietal microcirculation into the pleural space and is drained by lymphatics: the new feature is that flows, rather than pressure gradients, are presented in this figure in order to describe the pleural fluid turnover rate. The figure also shows that liquid filters from the pulmonary capillaries into the lung interstitium, from where it is removed by the lymphatics. The latter point also represents a new piece of knowledge [26], based strongly on interstitial pressure measurements performed with lungs physiologically expanded in the pleural space. The important concept to grasp here is that the lymphatics represent a drainage mechanism, able to generate a subatmospheric pressure (like a vacuum cleaner). From a mechanistic standpoint, the lymphatics set the pressure in the compartment to be drained; therefore, they establish an important variable appearing in the Starling pressure balance equation, namely interstitial hydraulic pressure.

As mentioned previously, similar concepts for fluid turnover are now also being shared for other interstitia and serous cavities [4], in fact, such a system allows a close control on interstitial volume and protein concentration. It is worth recalling here that when lungs are physiologically expanded in the chest, alveolar pressure is equal to atmospheric and pleural pressure is subatmospheric, so that, from the mechanical standpoint, the lung interstitium is subject to a tensile stress. Note that, if pulmonary interstital pressure were to be measured in isolated lungs passively inflated at positive alveolar pressure, a compressive stress would be applied to the lung tissue and this may raise interstitial pressure above atmospheric; clearly, such data bear little relationship to the physiological situation. Finally, as can be appreciated from the available database, it is clear that no reabsorption of pleural fluid can occur into the pulmonary capillaries, as hypothesized by NeERGARD [1] in 1927. In man no database is available to attempt an analysis of fluid turnover in the pleuropulmonary compartments; nevertheless, although numbers may vary somewhat relative to other species, the situation is unlikely to be qualitatively different based on anatomofunctional considerations and on data gathered in large mammals.

\section{Control and pathophysiology}

Another implication of the original hypothesis of NEERGARD [1] was that pleural liquid volume and protein concentration should vary on changing the Starling balance of pressure across the pleurae. However, both experimental evidence and medical practice indicate that pleural fluid volume and composition are highly stable and, in fact, pleural effusions develop when dramatic changes in fluid and solute homeostasis occur. Therefore, this suggests that some sort of mechanism ought to exist to guarantee a tight control on pleural fluid volume and protein concentration. The same happens, of course, in other tissues, although the features of the control vary.

In the lung parenchyma, for example, a very tight control exists to guarantee a minimum amount of interstitial fluid $[26,29]$ which is crucial to assure gas diffusion. The comparison between pleural space and lung interstitium offers, in fact, an important clue to the understanding of how a condition of minimum interstitial volume is achieved. The pulmonary interstitium has a very low mechanical compliance; accordingly, when facing a condition of increased filtration, this leads to a marked increase in pulmonary interstitial pressure [29]. This represents the so-called "tissue safety factor" against the development of pulmonary oedema, as it opposes, based on the Starling balance of pressures, a further filtration. However, due to its high compliance, the pleural space has no "tissue safety factor"; therefore, the only mechanism assuring a control on minimum pleural liquid volume is represented by lymphatic drainage. Since pleural lymphatics can increase the flow in response to an increase in pleural liquid volume, they represent a negative feedback mechanism controlling pleural liquid volume, as they tend to offset the induced perturbation

Such a system seems to be highly efficient. In fact, relatively wide variations in pleural filtration rate result

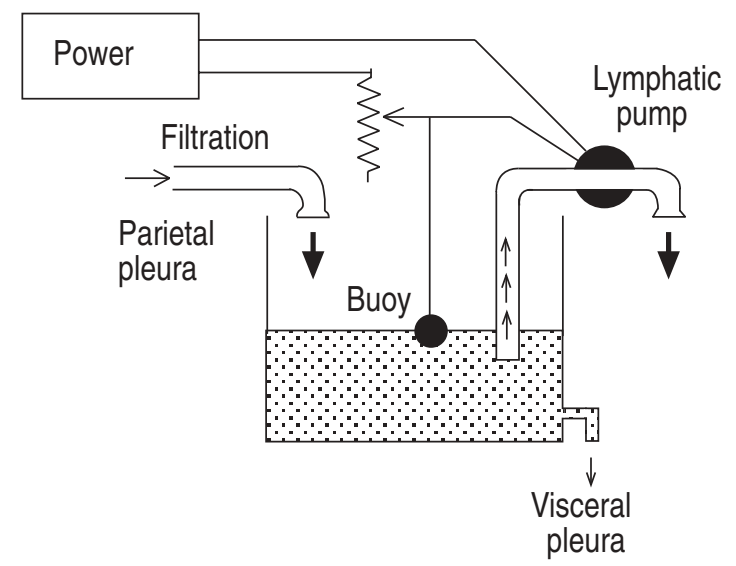

Fig. 3. - Simple schema to depict the features of lymphatic control. Lymphatics act as a pump controlled by a rheostat that can increase flow rate in response to an increase in pleural liquid volume. When the level of the buoy rises, the power to the pump increases and, correspondingly, the lymph flow increases also. 
in minor deviations from steady state pleural liquid volume [30], the latter being maintained at a minimum value. Figure 3 shows a simple functional schema of the pleural space and of pleural lymphatics. The visceral pleura is shown as a high resistance pathway as opposed to the parietal pleura and to the parietal lymphatics. Parietal lymphatics act as a pump controlled by a rheostat; when the level of the buoy rises (as a consequence of an increased filtration), the power to the pump increases and, correspondingly, lymph flow increases also.

To give an idea of such a regulatory mechanism, one may consider that for a 10 fold increase in filtration rate, the steady state pleural liquid volume would only increase by $15-20 \%$ [30], undetectable by common X-rays. The same regulatory capacity is retained even if the maximum lymph flow rate is decreased 10 fold. It must be emphasized, however, that the increase in lymph flow to attain its maximum (about 20 fold) is smaller than the potential increase in Starling dependent filtration flows. In fact, an increase in filtration rate by two orders of magnitude may result from pathological conditions (e.g. inflammation, right heart failure); clearly, an increase in pleural filtration rate beyond the maximum pleural lymph flow results in pleural effusion. Thus, although lymph flow may increase substantially, lymphatics cannot cope with a massive increase in filtration rate. It appears, therefore, more appropriate to regard lymphatics as a system mostly effective in controlling pleural liquid volume close to steady state conditions.

These conclusions may be valid in humans too, although some reservation is due because of the extrapolation. Let us consider a basal pleural lymph flow rate equal to that measured in dogs $\left(0.02 \mathrm{~mL} \cdot \mathrm{kg}^{-1} \cdot \mathrm{h}^{-1}\right.$ : for a $70 \mathrm{~kg}$ man this would correspond to $1.4 \mathrm{~mL}^{-1}$ or about 34 $\mathrm{mL} \cdot$ day $^{-1}$, about $2 \%$ of the overall daily lymphatic flow $\left(1 \mathrm{~mL} \cdot \mathrm{kg}^{-1} \cdot \mathrm{h}^{-1}\right.$ or $\left.\left.1,680 \mathrm{~mL} \cdot \mathrm{day}^{-1}\right)\right)$. Maximum pleural lymph flow would amount to $\approx 30 \mathrm{~mL} \cdot \mathrm{h}^{-1}, \approx 700 \mathrm{~mL} \cdot \mathrm{day}^{-1}$ (about $40 \%$ of overall lymph flow), a remarkable increase, and yet, still not enough to counteract the formation of large pleural effusions. Beyond maximum lymph flow saturation, fluid exchanges depend upon hydraulic and osmotic pressure gradients, whilst below saturation level, lymph flow represents the main drainage pathway. It seems appropriate to recall here the long overlooked hypothesis proposed by Starling 100 yrs ago concerning the fluid reabsorption from experimental pleural effusion, namely, that after hydraulic and osmotic equilibrium is accomplished, "...the absorption of fluid from the pleural cavity is extremely slow, so that it might perhaps be affected by the lymphatics alone" [31].

Based on the fact that pleural fluid is filtered and mostly reabsorbed via lymphatics at parietal level, an attempt was made to model pleural fluid turnover [32], as occurring between three compartments in series (systemic capillaries, extrapleural parietal interstitium and pleural cavity), separated by two resistances in series (capillary endothelium and parietal mesothelium), and two draining pathways (extrapleural and pleural lymphatics). As already mentioned, the visceral pleura does not appreciably account for pleural fluid egress under normal conditions. The model is useful to discuss some important determinants of pleural effusion. A simultaneous increase of capillary and mesothelial water permeability leads to hypo-oncotic fluid; if filtration exceeds maximum lymph flow, transudate would then form. Exudate would mostly form when protein permeability of the systemic capillaries is increased; a comparable increase in mesothelial protein permeability would only cause a modest increase in pleural liquid protein concentration because interstitial protein concentration is low already. Again, pleural effusion of exudate type would occur when filtration rate exceeds maximum lymph flow. It must be remembered that on biophysical grounds an increase in solute, compared to water permeability, reflects a more severe lesion of the membrane. Finally, it must be noted that the extrapleural interstitium exerts a potent buffer action against the increase in pleural filtration rate; indeed, due to its low compliance, an increased capillary filtration leads to a marked increase in interstitial pressure, thus opposing further capillary filtration.

\section{Pleural space and lung pathophysiology}

Some controversies should be reported concerning the permeability of the visceral pleura. Results from studies on stripped specimens of the visceral pleura [33] suggest that permeability of the visceral pleura to water and solutes is fairly high. Contrary to these conclusions are the results from experiments where the integrity of the pleural space and spontaneous respiration were preserved; in these conditions, it was found that albumin transport from pleural space to pulmonary interstitium accounted for less than $20 \%$ (an overestimate) of total albumin removal from the pleural space with close to normal pleural liquid volume [23]. Thus, the finding of a high permeability of the visceral pleura may suggest that experimental damage was induced. In line with the hypothesis of a highly permeable visceral pleura, other data [34] suggest that the visceral pleura may represent a pathway for fluid removal during pulmonary oedema; these data are based on the observation that protein rich liquid leaks through the visceral pleura of isolated, inflated, perfused lungs made oedematous in an artificial pleural space. Again, the finding, of a protein-rich liquid suggests a marked alteration in membrane permability both of capillaries and visceral pleura, because normal pleural fluid is hypo-oncotic.

Extrapolation of such findings to human pathology may be difficult, as protein-rich pleural effusion is a rather uncommon finding in pulmonary oedema. In fact, it was also found that during lung interstitial oedema in rabbits (that have a thin visceral pleura), pulmonary interstitial pressure rose well above atmospheric [29], creating a gradient for liquid filtration from the lung parenchyma into the pleural space. However, this did not result (at least up to $3 \mathrm{~h}$ of observation) in any appreciable increase in pleural liquid volume, indicating that the permeability of the visceral pleura is physiologically fairly low. Human clinical experience reveals that transudative pleural effusion may develop several hours after pulmonary oedema. To explain an increase in microvascular filtration through the visceral pleura, two hypotheses may be put forward: 1) on biophysical grounds, pulmonary vascular congestion implies an increase in surface area for microvascular exchanges (this may involve not only the pulmonary circulation but also the bronchial circulation that supplies the visceral pleura); 
and 2) water permeability of the visceral pleura increases as a general result of inflammation.

\section{Intrapleural fluid dynamics}

Intrapleural fluid movements were demonstrated by following the intrapleural distribution of radioactive albumin with a gamma camera $[18,23,38,35]$. Intrapleural flows have been depicted with a porous flow model, and the hydraulic resistivity of the pleural space was found to be about 5 orders of magnitude lower compared to an interstitial tissue [35]. Recently, evidence of recirculation of pleural fluid has been provided [36], and, furthermore, a model has been presented to account for transient intrapleural flows due to the effect of buoyancy of the lung into the pleural fluid [37]; the latter effect, reflecting a lower lung density relative to pleural fluid, would be relevant during dynamic changes relating to change in posture.

Figure 4 summarizes the major features of fluid turnover at pleural level. Filtration decreases going cephalad to caudad. Comparative studies confirm such a finding [38]. Conversely, lymphatic flow increases going cau$\mathrm{dad}$, and is also mainly localized to diaphragmatic and mediastinal surface. Finally, intrapleural flows drive fluid cephalad to caudad and towards diaphragmatic and mediastinal surfaces.

A final word on lubrication between sliding pleural membranes is necessary. The main function of the pleural fluid is to guarantee a close apposition of the visceral and pleural membranes, providing a frictionless sliding of such membranes during breathing. Oligolamellar sufactant molecules were demonstrated to be stratified on top of microvilli [39]. Since surfactant molecules are charged, they repulse each other on opposite surfaces; this would assure a graphite-like lubrication.

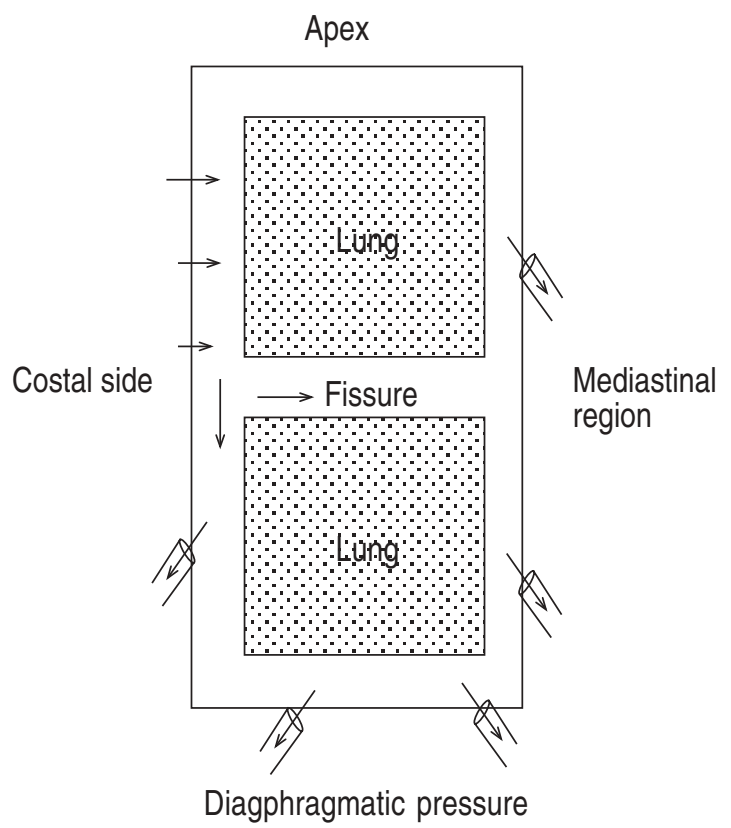

Fig. 4. - Overall functional arrangement of pleural flows. The arrows show: 1) that pleural liquid filtration decreases going from apex to base of the lung; 2) that pleural lymphatic flow is mostly localized on the diaphragmatic and mediastinal surface; and 3) the occurrence of intrapleural flows in the horizontal direction and from costal to mediastinal regions.

\section{References}

1. Neergard K. Zur Frage des Drukes in Pleuraspalt. Beitr Klin Erforsch Tuberk Lungenkr 1927; 65; 476-485.

2. Starling EH, Tubby AH. On absorption from and secretion into the serous cavities. J Physiol 1894; 16: 140155.

3. Kedem O, Katchalsky A. Thermodynamic analysis of the permeability of biological membranes to nonelecrolytes. Biochim Biophys Acta 1958; 45: 229-246.

4. Michel CC. Fluid movements through capillary walls. In: Renkin EM, Michel CC, eds. Handbook of Physiology. The Cardiovascular System: Microcirculation. Section 2, Vol IV, Part 1, Chapter 9. The American Physiological Society, Bethesda, MD, 1984; pp. 375-409.

5. Miserocchi G. Pleural pressures and fluid transport. In: Crystal RG, West JB, ed. The Lung: Scientific Foundations. Chapter 5.1.1.7. New York, Raven Press, 1991; pp. 885-893.

6. Miserocchi G. Agostoni E. Contents of the pleural space. J Appl Physiol 1971; 30: 208-218.

7. Negrini D. Integration of capillary, interstitial and lymphatic function in the pleural space. In: Reed RK, McHale NG, Bert JL, Winlove CP, Laine GA, eds. Interstitium Connective Tissues and Lymphatics. London, Portland Press, 1995; pp 283-299.

8. Bettendorf U. Electronmicroscopic studies on the peritoneal reabsorption of intrapleurally injected latex particles via the diaphragmatic lymphatics. Lymphology 1979; 12: 66-70.

9. Leak LV, Rahil K. Permeability of the diaphragmatic mesothelium: the ultrastructural basis for "stomata". Am J Anat 1978; 151: 557-594.

10. Negrini D, Mukenge S, Del Fabbro M, Gonano C, Miserocchi G. Distribution of diaphragmatic lymphatic stomata. J Appl Physiol 1991; 72: 1544-1549.

11. Wang NS. Mesothelial cells in situ. In: Chretien J, Bignon J, Hirsh A, eds. The Pleura in Health and Disease. New York, Marcel Dekker Inc., 1985 pp. 23-42.

12. Negrini D, Del Fabbro M, Gonano C, Mukenge S. Distribution of diaphragmatic lymphatic lacunae. J Appl Physiol 1992; 72: 1166-1172.

13. Albertine KH, Wiener-Kronish JP, Staub NC. The structure of the parietal pleura and its relationship to pleural liquid dynamics in sheep. Anat Rec 1984; 208: 401-409.

14. Mariassy AT, Wheeldon EB. The pleura: a combined light microscopy, scanning and transmission electron microscopic study in the sheep. Exp Lung Res 1983; 4: 293-313.

15. Bernaudin JF, Fleury J. Anatomy of the blood and lymphatics circulation of the pleural serosa. In: Chretien J, Bignon J, Hirsh A, eds. The Pleura in Health and Disease. New York, Marcel Dekker Inc., 1985; pp. 101-124.

16. Albertine KH, Wiener-Kronish JP, Roos PJ, Staub NC. Structure, blood supply and lymphatic vessels of the sheep's visceral pleura. Am J Anat 1982; 165: 277-294.

17. Agostoni E, Taglietti A, Setnikar I. Absorption of the capillaries of the visceral pleura in determination of interpleural pressure. Am J Physiol 1957; 191: 277-282.

18. Miserocchi G, Pistolesi M, Miniati M, Bellina CR, Negrini D, Giuntilli C. Pleural liquid pressure gradients and intrapleural distribution of injected bolus. $J$ Appl Physiol: Respirat Environ Exercise Physiol 1984: 56; 526-532.

19. Negrini D, Capelli C, Morini M, Miserocchi, G. Gravitydependent distribution of parietal subpleural interstitial pressure. J Appl Physiol 1987; 63: 1912-1918. 
20. Miserocchi G, Nearini D. Contribution of Starling and lymphatic flows to pleural liquid exchange in anesthetized rabbits. J Appl Physiol 1986; 61: 325-330.

21. Miserocchi G, Negrini D, Mukenge S, Turconi P, Del Fabbro M. Liquid drainage through the peritoneal diaphragmatic surface. J Appl Physiol 1989; 66: 1579-1585.

22. Negrini D, Ballard ST, Benoit JN. Contribution of lymphatic myogenic activity and of respiratory movements to pleural lymph flow. J Appl Physiol 1994; 76: 22672274.

23. Negrini D, Pistolesi M, Miniati M, Bellina CR, Giuntilli C, Miserocchi G. Regional protein absorption rates from the pleural cavity in dogs. $J$ Appl Physiol 1985; 58: 2062-2067.

24. Broaddus, VC, Wiener-Kronish JP, Berthiaume Y, Staub NC. Removal of pleural liquid and protein by lymphatics in awake sheep. J Appl Physiol 1988; 56: 384-390.

25. Wiener Kronish JP, Albertine KH, Roos PJ, Staub NC. Protein egress and entry rates in pleural fluid and plasma in sheep. J Appl Physiol: Respirat Environ Exercise Physiol 1984; 56: 459-463.

26. Miserocchi G, Negrini D, Gonano C. Direct measurements of interstitial pulmonary pressure in in situ lung with intact pleural space. J Appl Physiol 1990; 69: 2168-2174.

27. Negrini D, Gonano C, Miserocchi G. Microvascular pressure profile in intact in situ lung. J Appl Physiol 1992; 72: 332-339.

28. Miserocchi G, Negrini D, Pistolesi M, et al. Intrapleural liquid flow down a gravity-dependent hydraulic pressure gradient. J Appl Physiol 1988; 64: 577-584.

29. Miserocchi G, Negrini D, Del Fabbro M, Venturoli D. Pulmonary interstitial pressure in intact in situ lung: the transition to interstitial edema. J Appl Physiol 1993; 74: 1171-1177.

30. Miserocchi G, Venturoli D, Negrini D, Del Fabbro M. Model of pleural fluid turnover. J Appl Physiol 1993; 75: 1798-1806.

31. Leathes JB, Starlin EH. The absorption of salt solution from the pleural cavities. J Physiol 1895; 18: 106-116.

32. Venturoli D. Modello di scambio di liquido e soluti in un sistema a tre compartimenti: applicazione al turnover di liquido e soluti nel cavo pleurico. PhD Dissertation, University of Milano, 1995.

33. Paine DK, Kinasewitz GT, Gonzales E. Comparative permeability of canine visceral and parietal pleura. $J$ Appl Physiol 1988; 65: 2558-2564.

34. Broaddus VC, Wiener-Kronish JP, Staub NC. Clearance of lung edema into the pleural space of volume-loaded, anesthetized sheep. J Appl Physiol 1990; 68: 2623-2630.

35. Miserocchi G, Venturoli D, Negrini D, Gilardi MC, Bellina RC. Intrapleural fluid movements described by a porous flow model. J Appl Physiol 1992; 73: 25112516.

36. Butler JP, Huang J, Loring SH, Lai-Fook SJ, Wang PM, Wilson TA. Model of a pump that drives circulation of pleural fluid. J Appl Physiol 1995; 78: 23-29.

37. Grotberg JB, Glucksberg MR. A buoyancy-driven squeeze film model of intrapleural fluid dynamics: basic concepts. J Appl Physiol 1994; 77: 1555-1561.

38. Negrini D, Miserocchi G. Size-related differences in parietal extrapleural and pleural liquid pressure distribution. J Appl Physiol 1989; 67: 1967-1972.

39. Hills BA. Graphite-like lubrication of mesothelium by oligolamellar pleural surfactant. J Appl Physiol 1992; 73: 1034-1039. 